\title{
Corrigendum
}

\section{A far-infrared survey of bow shocks and detached shells around AGB stars and red supergiants}

\author{
N. L. J. Cox ${ }^{1}$, F. Kerschbaum ${ }^{2}$, A. J. van Marle $^{3}$, L. Decin ${ }^{1,4}$, D. Ladjal ${ }^{7}$, A. Mayer ${ }^{2}$, M. A. T. Groenewegen ${ }^{5}$,

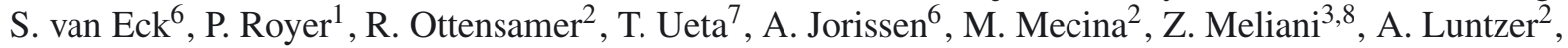 \\ J. A. D. L. Blommaert ${ }^{1}$, Th. Posch ${ }^{2}$, B. Vandenbussche ${ }^{1}$, and C. Waelkens ${ }^{1}$ \\ 1 Instituut voor Sterrenkunde, KU Leuven, Celestijnenlaan 200D, 3001 Leuven, Belgium \\ e-mail: nick@ster.kuleuven.be \\ ${ }^{2}$ University of Vienna, Department of Astronomy, Türkenschanzstraße 17, 1180 Wien, Austria \\ 3 Centre for Plasma-astrophysics, K.U.Leuven, Celestijnenlaan 200B, 3001 Leuven, Belgium \\ ${ }^{4}$ Sterrenkundig Instituut Anton Pannekoek, Universiteit van Amsterdam, Science Park 904, 1098 Amsterdam, The Netherlands \\ 5 Royal Observatory of Belgium, Ringlaan 3, 1180 Brussels, Belgium \\ 6 Institut d'Astronomie et d'Astrophysique, Université Libre de Bruxelles, CP 226, Boulevard du Triomphe, 1050 Bruxelles, Belgium \\ 7 Department of Physics and Astronomy, University of Denver, 2112 E. Wesley Ave., Denver, CO 80208, USA \\ ${ }^{8}$ Laboratoire Univers et Théories, Observatoire de Paris, UMR 8102 du CNRS, Université Paris Diderot, 92190 Meudon, France
}

A\&A 537, A35 (2012), DOI: 10.1051/0004-6361/201117910

Key words. stars: AGB and post-AGB - circumstellar matter - infrared: ISM - hydrodynamics - stars: mass-loss - errata, addenda

Two errors have appeared in the original published version of this article. A copy-and-paste error resulted in two subsequent LSR space motion corrections to the values for CW Leo reported in the original Table 1 . The correct values are given here in Table 1. The second error resulted from the omission of the "square" term for the distance in the actual computation of the dust mass following Eq. (5) $\left(M_{\text {dust }}=\frac{d^{2} F_{v}}{\kappa(v) B_{v}(T)}\right)$. This correction results in a, distance dependent, increase of dust mass by approximately two orders of magnitude. The complete corrected original Table 5 is reproduced in this erratum. Only the values in Cols. 9 and 10 have changed.

The above corrections give total observed gas and dust masses ranging from 0.002 to $0.56 M_{\odot}$. These corrections change in part the conclusions arrived at in the final paragraph of Sect. 3.3. In particular, we note that the inferred total mass of gas and dust is significantly higher than the potential mass swept-up from interstellar medium (ISM). The estimated swept-up ISM mass, $M_{\text {ISM }}$, ranges from $\sim 10^{-5}$ to $10^{-1} M_{\odot}$. These results indicate that on average only a few percent of the observed dust in the bow shock region originates from the surrounding medium. For individual objects, notably CW Leo and $\alpha$ Ori, the contribution from ISM dust could be as much as $10-25 \%$, while for others it is estimated to be even less than one percent (Table 5). These corrections now show that the derived masses are consistent with dust emission being predominantly from stellar wind grains trapped in the wind-ISM interaction rather than from
Table 1. LSR proper motion, space velocity, position angle and inclination for CW Leo.

\begin{tabular}{lcccc}
\hline \hline Target & $\begin{array}{c}\mu \\
\left(\mathrm{mas} \mathrm{yr}^{-1}\right)\end{array}$ & $\begin{array}{c}v_{\star} \\
\left(\mathrm{km} \mathrm{s}^{-1}\right)\end{array}$ & $\begin{array}{c}\text { PA } \\
\left({ }^{\circ}\right)\end{array}$ & $\begin{array}{c}i \\
\left({ }^{\circ}\right)\end{array}$ \\
\hline CW Leo & 53.1 & $41.0 \mathrm{~km} \mathrm{~s}^{-1}$ & 64.5 & -37.4 \\
\hline
\end{tabular}

interstellar grains, which is consistent with hydrodynamical modeling as discussed recently for $\alpha$ Ori by e.g. Mohamed et al. (2012) and Mackey et al. (2012). Note that the derived dust masses are sensitive not only to the adopted dust temperature, but also the dust opacity law, and the adopted gas-to-dust ratio. These uncertainties together potentially introduce an order of magnitude uncertainty to the derived total dust and gas mass. For example, an increase in dust temperature results in a decrease of the dust mass, thus increasing the relative importance of the ISM contribution.

Acknowledgements. We thank Dr. Anthony Marston for alerting us to the error in the dust mass calculation.

\section{References}

Harper, G. M., Brown, A., \& Guinan, E. F. 2008, AJ, 135, 1430

Mackey, J., Mohamed, S., Neilson, H. R., Langer, N., \& Meyer, D. M.-A. 2012, ApJ, 751, L10

Mohamed, S., Mackey, J., \& Langer, N. 2012, A\&A, 541, A1 
A\&A 543, C1 (2012)

Table 5. Aperture flux of observed bow shocks (Class I and II) and detached rings (Class III).

\begin{tabular}{|c|c|c|c|c|c|c|c|c|c|c|c|}
\hline \multirow[t]{2}{*}{ IRAS id } & \multirow[t]{2}{*}{ Object } & \multirow[t]{2}{*}{ Class } & \multicolumn{2}{|c|}{ Radius $^{a}$} & \multirow{2}{*}{$\begin{array}{l}\text { Dust annulus } \\
\left({ }^{\prime \prime}\right)\end{array}$} & \multicolumn{2}{|c|}{ Flux (Jy) } & \multicolumn{2}{|c|}{$M_{\text {dust }+ \text { gas }}^{b}\left(10^{-2} M_{\odot}\right)$} & \multirow{2}{*}{$\begin{array}{c}M_{\mathrm{ISM}^{c}} \\
\left(10^{-4} M_{\odot}\right)\end{array}$} & \multirow{2}{*}{$\begin{array}{c}M_{\mathrm{ISM}^{d}} \\
\left(10^{-4} M_{\odot}\right)\end{array}$} \\
\hline & & & $\left({ }^{\prime \prime}\right)$ & (pc) & & $70 \mu \mathrm{m}$ & $160 \mu \mathrm{m}$ & $70 \mu \mathrm{m}$ & $160 \mu \mathrm{m}$ & & \\
\hline $00248+3518$ & AQ And & I+III & 52 & 0.21 & $40-62$ (circle) & $2.20 \pm 0.01$ & $1.28 \pm 0.01$ & 19.4 & 16.3 & 1.1 & 4.4 \\
\hline $01159+7220$ & S Cas & III & $50^{e}$ & 0.23 & $32-64$ & $2.26 \pm 0.01$ & $1.11 \pm 0.02$ & 26.0 & 18.5 & 12 & 54 \\
\hline 01246-3248 & $\mathrm{R} \mathrm{Scl}$ & $\begin{array}{c}\text { I } \\
\text { III }\end{array}$ & $\begin{array}{l}54^{j} \\
14^{f}\end{array}$ & $\begin{array}{l}0.10 \\
0.03\end{array}$ & $51-68(\operatorname{arc})$ & $1.27 \pm 0.01$ & $0.49 \pm 0.01$ & 1.4 & 0.8 & 0.1 & 0.3 \\
\hline 02168-0312 & $o$ Cet & I & $82^{j}$ & 0.04 & $70-150(\operatorname{arc})$ & $47.64 \pm 0.03$ & $9.75 \pm 0.05$ & 5.2 & 1.5 & 0.5 & 0.2 \\
\hline $03374+6229$ & U Cam & III & $7^{f}$ & 0.02 & & & & & & & \\
\hline $03507+1115$ & NML Tau & $\begin{array}{l}\text { II } \\
\text { I }\end{array}$ & $\begin{array}{c}57 / 62^{i} \\
85\end{array}$ & $\begin{array}{c}0.12 / 0.13 \\
0.10\end{array}$ & $\begin{array}{c}80-140 \\
95-130(\operatorname{arc})\end{array}$ & $\begin{array}{l}2.87 \pm 0.02 \\
5.23 \pm 0.02\end{array}$ & $\begin{array}{l}2.44 \pm 0.05 \\
3.04 \pm 0.03\end{array}$ & $\begin{array}{l}6.9 \\
4.1\end{array}$ & $\begin{array}{l}8.5 \\
3.4\end{array}$ & $\begin{array}{l}40 \\
3.5\end{array}$ & $\begin{array}{c}1110 \\
62\end{array}$ \\
\hline $04459+6804$ & ST Cam & II & $67 / 84^{i}$ & $0.14 / 0.17$ & $84-122$ & $1.28 \pm 0.02$ & $0.61 \pm 0.03$ & 2.9 & 2.0 & 3.1 & 2.2 \\
\hline $05028+0106$ & W Ori & $\mathrm{I} / \mathrm{III}$ & 92 & 0.17 & $70-120$ & $1.99 \pm 0.02$ & $0.62 \pm 0.03$ & 3.7 & 1.7 & 8.2 & 32 \\
\hline $05418-4628$ & W Pic & I & $34^{j}$ & 0.08 & $62-90$ & $0.78 \pm 0.01$ & $0.25 \pm 0.02$ & 2.6 & 1.2 & 2.7 & 13 \\
\hline $05524+0723$ & $\alpha$ Ori $^{l}$ & I & $397^{j}$ & 0.25 & 510-660 (arc) & $56.68 \pm 0.19$ & $22.64 \pm 0.38$ & 28.5 & 16.5 & 64 & 155 \\
\hline $06331+3829$ & UU Aur & I & $82^{j}$ & 0.14 & $100-140(\operatorname{arc})$ & $5.44 \pm 0.02$ & $2.50 \pm 0.03$ & 8.2 & 5.4 & 14 & 88 \\
\hline $09448+1139$ & R Leo & I & $93^{j}$ & 0.03 & 94-134 & $6.31 \pm 0.02$ & $1.92 \pm 0.03$ & 0.4 & 0.2 & 0.1 & 2.1 \\
\hline $09452+1330$ & CW Leo ${ }^{k}$ & I & $507^{j}$ & 0.29 & 560-710 (arc) & $6.88 \pm 0.08$ & $\begin{array}{c}11.13 \pm 0.11 \\
6.00 \pm 0.13\end{array}$ & 1.3 & $\begin{array}{l}3.0 \\
1.6\end{array}$ & 76 & 31 \\
\hline $10329-3918$ & U Ant & III & 42 & 0.06 & $30-55$ (circle) & $16.32 \pm 0.01$ & $4.68 \pm 0.01$ & 15.2 & 6.3 & 0.4 & 5.3 \\
\hline $10350-1307$ & U Hya & $\mathrm{I}+\mathrm{III}$ & 114 & 0.12 & $100-133$ (circle) & $17.44 \pm 0.03$ & $9.33 \pm 0.03$ & 9.8 & 7.6 & 1.7 & 0.03 \\
\hline $10416+6740$ & VY UMa & II & $38 / 46^{i}$ & $0.07 / 0.09$ & $38-88$ & $3.60 \pm 0.01$ & $2.33 \pm 0.02$ & 6.7 & 6.3 & 0.7 & 2.5 \\
\hline $10580-1803$ & R Crt & II & $\sim 140$ & 0.18 & $165-270$ & $5.07 \pm 0.07$ & $6.42 \pm 0.11$ & 4.5 & 8.2 & 21 & 58 \\
\hline $12427+4542$ & Y CVn & III & $\sim 190$ & 0.30 & $150-260$ & $5.14 \pm 0.07$ & $3.79 \pm 0.10$ & 6.9 & 7.3 & 7.7 & 2.9 \\
\hline $13001+0527$ & RT Vir & I & & & 50-140 (circle) & $5.15 \pm 0.03$ & $3.30 \pm 0.04$ & 1.2 & 1.1 & 0.6 & \\
\hline $13269-2301$ & R Hya & I & $96^{j}$ & 0.05 & $200-245$ (arc) & $4.65 \pm 0.02$ & $1.93 \pm 0.03$ & 0.9 & 0.6 & 3.8 & 12 \\
\hline $13462-2807$ & W Hya & III & $68,230^{g}$ & $0.03,0.12$ & $70-108$ (ellipse) & $21.28 \pm 0.02$ & $6.08 \pm 0.03$ & 3.0 & 1.2 & 0.2 & 0.1 \\
\hline $14003-7633$ & $\theta$ Aps & I & $76^{j}$ & 0.04 & $118-146(\operatorname{arc})$ & $2.15 \pm 0.01$ & $0.76 \pm 0.02$ & 0.4 & 0.2 & 1.3 & 0.4 \\
\hline $15094-6953$ & X Tra & III & $150^{h}$ & 0.18 & $60-210$ & $9.70 \pm 0.08$ & $6.89 \pm 0.12$ & 16.3 & 16.7 & 106 & 21 \\
\hline $16011+4722$ & X Her & I & $45^{j}$ & 0.03 & 40-90 (ellipse) & $9.17 \pm 0.01$ & $3.23 \pm 0.02$ & 2.2 & 1.1 & 0.2 & 0.2 \\
\hline $17389-5742$ & V Pav & II & $97 / 100^{i}$ & $0.17 / 0.18$ & $95-140$ & $1.30 \pm 0.03$ & $1.50 \pm 0.04$ & 2.3 & 3.8 & 21 & 12 \\
\hline $18476-0758$ & S Sct & III & 70 & 0.13 & 30-90 (circle) & $14.09 \pm 0.02$ & $8.85 \pm 0.03$ & 27.2 & 24.7 & 13 & \\
\hline $19126+3247$ & W Aql & II & $45 / 75^{i}$ & $0.07 / 0.12$ & 36-86 (ellipse) & $11.10 \pm 0.02$ & $4.25 \pm 0.03$ & 16.6 & 9.2 & 5.8 & 144 \\
\hline $19233+7627$ & UX Dra & II & $76 / 54^{i}$ & $0.14 / 0.10$ & $50-110$ & $2.89 \pm 0.01$ & $1.11 \pm 0.03$ & 5.6 & 3.1 & 4.4 & 2.5 \\
\hline $19314-1629$ & AQ Sgr & I & 57 & 0.09 & $50-100$ & $3.44 \pm 0.02$ & $2.53 \pm 0.02$ & 4.9 & 5.3 & 5.4 & 3.7 \\
\hline $19390+3229$ & TT Cyg & III & 33 & 0.07 & $26-43$ (circle) & $1.74 \pm 0.01$ & $0.86 \pm 0.01$ & 4.3 & 3.1 & 1.3 & 21 \\
\hline $20038-2722$ & V1943 Sgr & I & $66^{j}$ & 0.06 & $50-130(\operatorname{arc})$ & $5.98 \pm 0.03$ & $2.30 \pm 0.03$ & 3.0 & 1.7 & 2.6 & 0.8 \\
\hline 20075-6005 & X Pav & I & $50^{j}$ & 0.07 & $94-122$ & $5.74 \pm 0.01$ & $2.69 \pm 0.02$ & 4.4 & 2.3 & 3.1 & 19 \\
\hline 20141-2128 & RT Cap & III & 92 & 0.13 & $62-118$ & $2.21 \pm 0.02$ & $0.91 \pm 0.04$ & 2.4 & 1.4 & 4.0 & 15 \\
\hline $20248-2825$ & T Mic & I & & & 40-100 (ellipse) & $5.48 \pm 0.02$ & $0.58 \pm 0.03$ & 3.2 & 0.5 & 1.2 & \\
\hline $21358+7823$ & S Cep & III & 90 & 0.18 & $70-130$ & $2.14 \pm 0.03$ & & 4.6 & & 11 & 142 \\
\hline $21419+5832$ & $\mu$ Cep & I & $78^{j}$ & 0.15 & $100-150$ & $28.64 \pm 0.04$ & $11.5 \pm 0.12$ & 56.4 & 32.8 & 56 & 637 \\
\hline $21439-0226$ & EP Aqr & I & $43^{j}$ & 0.03 & $45-68$ & $3.68 \pm 0.01$ & $0.99 \pm 0.01$ & 0.6 & 0.2 & 0.1 & 1.2 \\
\hline \multirow{2}{*}{$23438+0312$} & TX Psc & III & $16^{f}$ & 0.02 & & & & & & & \\
\hline & & I & $38^{j}$ & 0.05 & $12-58$ (ellipse) & $4.79 \pm 0.01$ & $1.07 \pm 0.02$ & 4.7 & 1.5 & 0.2 & 0.7 \\
\hline $23558+5106$ & R Cas & I & $97^{j}$ & 0.06 & $100-160(\operatorname{arc})$ & $8.35 \pm 0.01$ & $3.52 \pm 0.04$ & 1.7 & 1.1 & 2.5 & 2.9 \\
\hline
\end{tabular}

Notes. Irregular Class IV is excluded. ${ }^{(a)}$ Radii of rings and fermata derived from the azimuthally averaged radial profiles (Fig. 1). ${ }^{(b)}$ Derived from Eq. (1) using the total integrated flux at 70 and $160 \mu \mathrm{m}$, respectively, adopting a gas-to-dust ratio of 200 and a dust temperature of $30 \mathrm{~K} .{ }^{(c)} M_{\text {ISM }}=$ $\frac{4}{3} \pi r^{3} \rho_{\text {ISM }}$, with $n_{\mathrm{H}}$ taken from Eq. (5). ${ }^{(d)} M_{\mathrm{ISM}}$, with $n_{\mathrm{H}}$ taken from the local densities inferred from the measured stand-off distance and Eq. (2); Tables 1 and 2. ${ }^{(e)}$ Central source is offset by $0^{\prime \prime}$ RA, $5^{\prime \prime}$ Dec. ${ }^{(f)}$ Ring radius from the deconvolved image. ${ }^{(g)}$ Inner and outer (at $\left.160 \mu \mathrm{m}\right)$ rings, respectively. ${ }^{(h)}$ Central source is offset by $12^{\prime \prime}$ RA, $5^{\prime \prime}$ Dec. ${ }^{(i)}$ Radial distances are quoted for both north and south arcs (east-west for W Aql). ${ }^{(j)}$ Projected distance $A$ (Fig. 6). ${ }^{(k)} \mathrm{CW}$ Leo has been observed two times at $160 \mu \mathrm{m} .{ }^{(l)}$ Updated distance to 197 pc (Harper et al. 2008). 